\title{
28 Research Square \\ Zoledronic Acid Induces Early Changes of VEGF Levels in Women With Postmenopausal Osteoporosis
}

Federica Bellone ( $\nabla$ fbellone@unime.it )

University of Messina

Antonino Catalano

University of Messina

Angelo Ruggero Sottile

University of Messina

Agostino Gaudio

University of Catania

Saverio Loddo

University of Messina

Francesco Corica

University of Messina

Nunziata Morabito

University of Messina

\section{Research Article}

Keywords: Bisphosphonate, zoledronic acid, vascular endothelial growth factor, osteonecrosis, postmenopausal, osteoporosis

Posted Date: May 28th, 2021

DOI: https://doi.org/10.21203/rs.3.rs-530052/v1

License: (c) (1) This work is licensed under a Creative Commons Attribution 4.0 International License.

Read Full License 


\section{Abstract}

Zoledronic acid (Zol) is a widely used intravenous aminobisphosphonate to treat both benign and malignant skeletal diseases, and bisphosphonate-related osteonecrosis of the jaw (BRONJ) is a serious side effect whose pathophysiology remains poorly understood. Vascular Endothelial Growth Factor (VEGF) has been recognized to mediate BRONJ in cancer patients undergoing Zol treatment, however data on VEGF are lacking in patients with osteoporosis.

The aim of this study was to investigate the influence of Zol on VEGF levels in women with postmenopausal osteoporosis.

28 postmenopausal women with osteoporosis were enrolled and randomized into 2 groups to receive Zol (5 mg) or placebo. At baseline, at day-3 and day-30 VEGF serum levels were measured; bone turnover markers, 25-hydroxyvitamin D (25(OH)D) and serum calcium were evaluated at baseline.

In Zol-treated women, VEGF increased significantly on day-3, and then decreased on day-30. The VEGF change $(-18 \%$ at day- 30 vs. baseline, $p=0.01)$ was significantly associated with $25(\mathrm{OH}) \mathrm{D}$ levels, after correcting for age, BMI, time since menopause, femoral neck BMD, osteocalcin, C-terminal telopeptide of type 1 collagen and baseline VEGF levels.

For the first time, we detected early modifications of circulating VEGF in postmenopausal women receiving Zol for osteoporosis, identifying a vitamin D-dependent modulation of these changes.

\section{Introduction}

Zoledronic acid (Zol) is a powerful intravenous aminobisphosphonate (N-BP) currently used against primary or secondary osteoporosis [1]. As shown in a randomized, double-blind, placebo-controlled, multinational study, the HORIZON trial, when compared with the placebo control group, Zol $5 \mathrm{mg}$ administered once a year for up to 3 years was demonstrated to increase bone mineral density (BMD) at different skeletal regions, improve bone metabolism markers and reduce the risk of new morphometric vertebral and hip fractures [2,3]. Zol treatment is commonly safe and well tolerated, with the most frequent adverse events being transient and reversible [4].

Osteonecrosis of the jaw $(\mathrm{ONJ})$ represents an emergent severe oral disease, with dramatic implications for health related patient's quality of life [5]. Since 2003, there has been a surge of interest in the effects of BPs on oral health, and ONJ has been described as a BP related class effect, and the acronym "BRONJ" was introduced to indicate Bisphosphonate-Related Osteonecrosis of Jaw. The American Association of Oral and Maxillofacial Surgeons (AAOMS) defines BRONJ as an exposure of portions of the jawbone in patients who have been exposed to BPs, lasting more than eight weeks in patients with no history of jaw irradiation [6]. Nonetheless, recent studies have clearly shown that also the antiresorptive agent denosumab and some antiangiogenic drugs could similarly induce ONJ $[7,8]$. Accordingly, the term Medication Related Osteonecrosis of Jaw (MRONJ) has been also acknowledged in medical literature 
$[9,10]$. In metastatic cancer patients, the risk of developing BRONJ has been mainly associated with BP potency, high and cumulative dose as well as intravenous administration $[11,12]$. BRONJ has been rarely reported in osteoporotic postmenopausal women [13].

The pathophysiology of BRONJ is still uncertain and under debate. BPs act on a wide variety of cell types, possibly increasing susceptibility to infections in the oral cavity, and impairing mucosal healing by acting on monocyte differentiation, angiogenesis or both [14]. Several cases of MRONJ have been described in cancer patients treated with bevacizumab, a recombinant human monoclonal antibody that binds to Vascular Endothelial Growth Factor (VEGF) and inhibits angiogenesis [15]. In accordance with published data, angiogenesis suppression may play a role in the MRONJ pathogenesis. Beyond the known antiresorptive, immunomodulating and direct antitumor actions, BPs are actually considered also antiangiogenic drugs. Particularly, the anticancer properties of Zol could even depend by inhibition of tumour angiogenesis via the reduction of circulating VEGF [16]. Zol has been proven to decrease VEGF concentrations in patients with metastatic bone cancer [17]. Serum VEGF levels after the first administration of intravenous BPs has been proposed as a possible early predictive marker of BRONJ in cancer patients [18].

Postmenopausal women exhibit a higher risk of periodontal diseases due to the fall of oestrogen levels, which exert a trophic action on oral cavity [19]. Scardina et al. showed a decreased periodontal capillary density, an increased tortuosity and decreased diameter of loops in oral microcirculation in a setting of postmenopausal women [20]. Consequently, the oral microcirculatory alterations observed in postmenopause may encourage the deleterious effects of some medications, such as Zol, on oral health.

It is still unclear whether a therapeutic dose of Zol for the medical management of postmenopausal osteoporosis have anti-angiogenic effects as previously recognized in cancer patients. The primary aim of this study was to explore the in vivo effect of Zol on VEGF levels in a cohort of postmenopausal women treated for osteoporosis.

\section{Methods}

\section{Study Subjects}

This prospective randomized placebo-controlled study considered a cohort of Caucasian postmenopausal women attending the Centre for Prevention, Diagnosis and Treatment of Osteoporosis in the Department of Internal Medicine of the University of Messina. Participants were consecutively recruited if affected by osteopenia or osteoporosis (defined in accordance with the WHO diagnostic criteria) with at least one prevalent vertebral fracture who required i.v. therapy with Zol in accordance with standards of good clinical practice. Exclusion criteria were moderate to severe chronic kidney or liver failure, secondary causes of osteoporosis including hyperthyroidism, hyperparathyroidism, hypercortisolism or malabsorption, in situ or systemic disease that could have impaired microcirculation such as diabetes mellitus, dyslipidemia, arterial hypertension, connective tissue diseases, autoimmune disorders or oral lichen planus. Patients undergoing treatment for osteoporosis (e.g., BPs, teriparatide, 
denosumab, selective estrogen receptor modulators, strontium ranelate and calcitonin) or with a history of prior therapy with these bone agents were also not considered.

Participants were enrolled after a careful clinical check including dental examination with preventive dentistry; women with poor oral hygiene, periodontal diseases, poorly fitting dentures, history of dental diseases, invasive dental procedures, e.g. tooth extractions, or with unhealed open soft tissue lesions in the mouth were not considered for this study.

Fracture risk was assessed by using Fracture Risk Assessment Tool (FRAX), a computer-based algorithm (http://www.shef.ac.uk/FRAX) that estimates the 10-year probability of a major fracture (hip, clinical spine, humerus, or wrist fracture) and the 10-year probability of a hip fracture, as previously reported [21]. All the patients were under vitamin D supplementation (cholecalciferol 25,000 IU every two weeks) and they took calcium (calcium carbonate 500 - 1000 mg daily) when needed to reach the recommended daily calcium intake.

Recruited women were randomized into two groups using a computer-generated randomization schedule in the order in which they were enrolled in the study.

At baseline, 18 participants underwent a single Zol $5 \mathrm{mg} / 100 \mathrm{~mL}$ (Aclasta ${ }^{\circledR}$ ) administration at 10.00 a.m. and within 30 minutes. 10 participants, not receiving Zol served as controls and received i.v. $100 \mathrm{~mL}$ saline solution $0.9 \%$.

The study was approved by our institutional research committee Policlinico "G. Martino" Messina (Prot.n. 71/19); it was performed in accordance with the 1964 Declaration of Helsinki and its later amendments for experiments involving humans and uniform requirements for manuscripts submitted to biomedical journals. Signed informed consent was obtained from all the participants.

\section{Biochemical Assays}

Serum samples were collected just before Zol administration at baseline, and then at day-3 and day-30 after Zol administration. Serum was separated from the blood corpuscles by centrifugation and stored frozen at $-80^{\circ} \mathrm{C}$ until analyzed.

VEGF-A serum levels were measured at each time-point by Enzyme-Linked Immunosorbent Assay, ELISA (Duoset, R\&D Systems Europe Ltd., Abingdon, UK) according to the manufacturer's instructions. The limit of detection of human VEGF-A defined as the analyte concentration resulting in an absorption significantly higher than that of the dilution medium (mean plus two standard deviations) was determined to be $7.9 \mathrm{pg} . / \mathrm{mL}$ (mean of 6 independent assays).

The inter- and intra-assay coefficient of variation was $4.3 \%$ and $6.2 \%$ respectively.

At baseline, levels of Osteocalcin (BGP), Bone-specific alkaline phosphatase (B-ALP) as markers of bone formation, and serum C-telopeptide of type 1 collagen (CTX), as marker of bone resorption, 25- 
hydroxyvitamin $\mathrm{D}(25(\mathrm{OH}) \mathrm{D})$ and serum calcium were recorded.

BGP was measured by immunoenzymatic assay (Invitrogen Ltd, UK) with an intra-assay coefficient of variance (CV) of $3.1 \%$ and inter-assay $\mathrm{CV}$ of $3.5 \%$; BSAP was measured by immunoenzymatic assay with the Access Immunoassay System (Beckman Coulter, Fullerton, California) with intra- and interassay CVs of $2.3 \%$ to $3.7 \%$ and $4.9 \%$ to $9.8 \%$, respectively; CTX was assessed using the Elecsys 2010 Immunoassay System (Roche, Basel, Switzerland) with intraassay CVs of $1.6 \%$ to $3 \%$ and interassay CVs of $1.3 \%$ to $4.3 \%$; levels of $25(\mathrm{OH}) \mathrm{D}$ were detected by high performance liquid chromatography; serum calcium was measured using standard laboratory techniques.

\section{Bone mineral density and vertebral fractures}

At baseline, a Dual-energy X-ray Absorptiometry (DXA) densitometer (Hologic Discovery) was used to assess BMD at the lumbar spine (L1-L4) and at the femoral neck. The DXA calibration was performed daily following the manufacturer's instruction. Its coefficient of variation (CV) was $0.5 \%$.

Vertebral fractures $(\mathrm{Vfs})$ were detected by vertebral morphometry at a lateral scan of thoracic and lumbar regions of the spine. Vfs was defined in accordance to the semiquantitative method proposed by Genant: a fracture was diagnosed if a vertebra body had at least a $20 \%$ height reduction in the anterior, middle or posterior height compared with the same or adjacent vertebra [22].

\section{Statistical analysis}

Statistical analyses were performed using MedCalc software (version 10.2.0.0; MedCalc Software, Mariakerke, 173 Belgium). Values were expressed as mean \pm SD. The normal distribution of values was verified with the Kolmogorov-Smirnov test. Differences were evaluated using the Student's t-test for paired and unpaired observations and repeated measures analysis of variances. Pearson's correlation coefficient was calculated to evaluate the degree of association between two variables. A multiple regression analysis was performed to determine the influence of one independent variable after correcting for the others. A post-hoc analysis was performed to explore change of VEGF levels in participants who experienced or not an Acute Phase Response (APR) after Zol administration. For all the tests, a P value of 0.05 or less was considered to indicate statistical significance.

\section{Results}

Baseline clinical features and main laboratory data of the participants are shown in Table 1. Particularly, at baseline, no significant differences in any of the investigated variables were observed between both groups of women.

In the overall population $(n=28)$, serum VEGF values were positively associated with femoral BMD $(r=$ $0.42 ; p<0.001)$ (Figure 1), and this correlation was maintained even after adjusting for age and BMI ( $r=$ $0.58 ; p=0.01)$. 
At baseline, VEGF values were associated with BGP levels ( $r=0.57 ; p<0.001)$ (Figure 2), and serum BGP positively correlated with femoral neck BMD $(r=0.33 ; p=0.003)$ (Figure 3 ).

In the 18 participants who received Zol, mean VEGF levels significantly increased at day-3 to a value of $138.83 \pm 100.89 \mathrm{pg} / \mathrm{mL}(+13 \%$ vs. baseline, $\mathrm{p}=0.04)$, and then decreased at day-30 to a value of $102.43 \pm$ $72.21 \mathrm{pg} / \mathrm{mL}$ (-26\% vs. day-3, $\mathrm{p}=0.002$ and $-18 \%$ vs. baseline, $\mathrm{p}=0.01)$ (Figure 4). Conversely, controls did not show any significant changes over time $(114.61 \pm 68.13 \mathrm{pg} / \mathrm{mL}$ and $110.72 \pm 71.12 \mathrm{pg} / \mathrm{mL}$ at day-3 and day-30, respectively; $\mathrm{p}_{\text {all }}>0.5 \mathrm{vs}$. baseline). In the Zol-treated women, the percent change of VEGF levels between baseline and day-30 was significantly associated with serum $25(\mathrm{OH}) \mathrm{D}$ values $(r=0.29, p=$ 0.028) (Figure 5). Change of VEGF at day-3 was also associated with CTX levels $(r=0.77, p=0.003)$. At a stepwise multiple regression analysis, 25(OH)D levels were the only predictor of VEGF change, after correcting for age, BMI, time since menopause, femoral neck BMD, BGP, CTX and baseline levels of VEGF $(\beta=1.7, S E=0.71, p=0.03)$. A post-hoc analysis showed that the rise of VEGF at day-3 was higher in the 3 participants who suffered APR in comparison with the other women receiving Zol who did not suffer APR [153.85 \pm 126.5 vs. $135.15 \pm 100.1(+13 \%, p=0.3)]$, although not reaching a statistical significance.

\section{Discussion}

Zol is the most potent and long-acting N-BP used for the treatment of osteoporosis, Paget's disease, myeloma and cancers to reduce adverse skeletal related events [1]. Change of VEGF levels have been previously reported in cancer patients receiving Zol [17], and decreased VEGF values have been also associated with the BRONJ risk [18].

To the best of our knowledge, this is the first prospective cohort study investigating the early serum changes of VEGF levels in postmenopausal women at high fracture risk receiving Zol. Particularly, VEGF levels significantly raised soon after 3 days from Zol administration, and then decreased after 30 days to a value below the baseline one.

The raise of VEGF at day-3 may fall in the window of the frequent occurring APR after i.v. N-BP administration.

APR is a common side effect observed in 10 to $50 \%$ of patients after the first Zol dose. It consists of a systemic immune reaction induced by the stimulation of circulating $\gamma \delta T$ cells and the subsequent release of several inflammatory cytokines as interleukin 6 (IL-6), tumor necrosis factor- $\beta$ (TNF- $\beta$ ) and interferon- $\gamma$ (IFN-ץ) $[23,24]$. Related symptoms occur within 24-36 hours and usually disappear in about 3 days [25].

VEGF is a specific endothelial cell mitogen and a strong vascular permeability factor; thus, it may be considered as a proinflammatory cytokine. Consequently, in patients receiving Zol, the early increase of VEGF levels could be a biochemical mark of VEGF involvement in the cytokine storm leading to APR [26]. Accordingly, at a post-hoc analysis, the rise of VEGF at day-3 was $13 \%$ higher in the 3 participants who suffered APR. 
On the other hand, the decrease of VEGF levels after 30 days could be determined by the well-known Zol related suppression of bone turnover and Zol activity on endothelial cells. Osteoclasts, osteoblasts, osteocytes and endothelial cells are sources of VEGF [27]. In the bone tissue, VEGF stimulates angiogenesis, osteoblast differentiation, and finally bone formation and bone healing. Furthermore, VEGF has been shown to promote osteoclast differentiation and survival [28]. Accordingly, VEGF could orchestrate the crosstalk between endothelial, osteoblastic and hematopoietic cells [29]. Data on VEGF levels in osteoporotic patients are conflicting, and circulating VEGF could be influenced by treatment [30].

In all the participants of this study, at baseline, we observed a significant positive association between VEGF and both BGP and femoral BMD. These findings have not been previously described and add new insights about the possible VEGF key role in bone health; moreover, they are consistent with previous studies showing significantly lower VEGF levels in women with postmenopausal osteoporosis [31].

Several lines of evidence based on both in vitro and in vivo studies [17, 18,32,33] suggest the antiangiogenic and anti-tumor VEGF-related Zol effect. However, few researches involved humans and mostly cancer patients. In 30 patients suffering from solid tumors with bone metastases, treated with a single infusion of Zol $4 \mathrm{mg}$, Santini et al. reported a significant reduction of VEGF levels after 2, 7 and 21 days, with the largest significance ( $p<0.001)$ on day 21 [34]. Moreover, low intermittent doses of Zol $(1 \mathrm{mg}$ every 7 days for 4 times followed by 4 mg every 28 days for 3 times), produced significant reductions of the circulating VEGF early after 7 days in cancer patients with bone metastasis from solid tumors. Moreover, after 84 days and a $16 \mathrm{mg}$ cumulative Zol dose, VEGF levels tended to rise, although remaining significantly lower in comparison with baseline [34]. Remarkably, even though the different dose and schedule, the reduction of serum VEGF levels observed in osteoporotic postmenopausal women in our study was of a smaller magnitude than that one seen in the oncology setting.

In women with postmenopausal osteoporosis, only one elegant study by Ishtiaq et al. evaluated the VEGF change in vivo and in vitro after N-BPs. Alendronate (Aln), administered at a weekly dose of $70 \mathrm{mg}$, produced a VEGF decline after 3 and 6 months, reaching a statistical significance after 12 months $(p=$ 0.02). Consistently, Aln and Zol, in the culture medium of two osteoblastic cell lines, significantly reduced the VEGF production, confirming that N-BP related in vivo modification of this cytokine should be attributed to a direct effect on bone cells [35].

The VEGF reduction obtained in vitro with Zol was more pronounced than that one with Aln and it occurred at a lower dose, in accordance with higher Zol potency [35]. Differently from N-BPs, no published data are available describing the denosumab effects on VEGF. Denosumab is a fully human monoclonal IgG2 antibody against the receptor activator of nuclear factor-kB ligand (RANKL) and it represents a potent antiresorptive agent for osteoporosis [36]. However, as for N-BPs, an antiangiogenic activity of denosumab, possibly mediated by a RANKL-dependent pathway, have been suggested by Girolami et al. [37].

Vitamin D has been proven to influence VEGF levels: calcitriol, the active vitamin D metabolite, promoted VEGF production, and by adding calcitriol to the medium in osteoblastic cell lines, the N-BP induced 
suppression of VEGF release was reverted [35]. Conversely, few discordant data exist, coming from breast cancer women treated with tamoxifen [38] and chronic spontaneous urticaria patients [39], both showing a significantly vitamin D-induced suppression of VEGF levels.

In line with the largest share of evidences, an inadequate vitamin D status could even amplify the VEGFmediated suppression of angiogenesis induced by N-BPs, and particularly by Zol, but it represents only a speculation, which needs to be confirmed by further focused researches.

Our findings are consistent with in vitro data by Ishtiaq et al. [35], although the significance of the effect of N-BPs on the production of pro-angiogenic factors to in vivo pharmacology remain uncertain. Moreover, the serum 25(OH)D concentration, indicative of vitamin D status, was significantly associated with the VEGF change in postmenopausal women receiving Zol. Although divergent data exist, the influence of vitamin D on VEGF levels is considered an extraskeletal vitamin D effect $[40,41]$.

Recent studies have shown an association between vitamin D deficiency and the risk BRONJ [42, 43]. An increased prevalence of BRONJ has been observed in vitamin D deficient rats exposed to Zol in comparison with vitamin D repleted rats [42]. Also in humans, one study showed that patients with secondary hyperparathyroidism due to hypovitaminosis $\mathrm{D}$, under N-BP treatment, were more likely to develop BRONJ [43]. Remarkably, in a case-control study, Bedogni and coauthors, at a histomorphometric analysis, observed that $77 \%$ of patients with BRONJ resulted osteomalacic compared with $5 \%$ of patients without BRONJ [44].

VEGF has been suggested as a potential biomarker for the development of BRONJ; in fact, Vincenzi et al. [18], in a cohort of 81 cancer patients receiving i.v. N-BPs, observed the development of BRONJ in six patients who showed the largest reduction of circulating VEGF levels after 7 and 21 days after the first NBP administration.

In light of these data, patients affected by Zol-induced ONJ, was effectively treated with platelet-rich plasma which represents a concentrate of growth factors, including VEGF, obtaining an improvement of the perilesional bone tropism [45].

We must recognize this study has limitations including the small sample size, consisting of only women in postmenopausal age and the observation period not long enough to capture Zol-induced VEGF modifications in the long term. This study is not focused on BRONJ; thus, the observed changes of VEGF levels after Zol administration may have uncertain significance, and their pathophysiological role requires additional investigations. Thus, further studies, focused on osteoporotic postmenopausal women, with a large sample size and with a long follow-up, are needed to confirm our data and to address the association with BRONJ. Additionally, measuring other angiogenic factors could lead to a more in-depth profile of the effect of Zol on angiogenesis.

At the same time, our findings improve the knowledge of cytokine modifications after N-BP administration, suggesting a role for vitamin D on the Zol mediated VEGF perturbancies. 
In conclusion, in osteoporotic postmenopausal women, Zol administration at a dose of $5 \mathrm{mg}$ provoked early modifications of circulating VEGF, and vitamin D has been shown to modulate this VEGF change. Since Zol-induced VEGF modifications could contribute to the pathogenesis of BRONJ, further studies focused on VEGF are urgently needed to investigate its role as an early marker of increased risk of BRONJ in patients treated for osteoporosis.

\section{Declarations}

\section{Data availability}

The datasets generated during and/or analysed during the current study are available from the corresponding author on reasonable request.

\section{Acknowledgements}

We thank our experienced technician, Santa De Salvo, for assistance in the assessment of bone mineral density at the lumbar spine and hip sites with our densitometer.

\section{Author contributions}

F.B., A.C., N.M. designed the study, A.R.S., A.C., F.B. analyzed data and wrote the manuscript. A.G., S.L.,

F.C. had full access to all the data in the study and take responsibility for the integrity and the accuracy of the

data analysis. All authors reviewed the manuscript.

\section{Funding}

This study did not receive external funding. None of the Authors received any funds to design and conduct the

study.

\section{Competing interests}

The authors declare no competing interests.

\section{References}

1. Reid, I.R. et al. Zoledronate. Bone137:115390, DOI: https://doi.org/10.1016/j.bone.2020.115390 (2020).

2. Black, D.M. et al; HORIZON Pivotal Fracture Trial. Once-yearly zoledronic acid for treatment of postmenopausal osteoporosis. N Engl J Med. 356(18):1809-1822 DOI: 
https://doi.org/10.1056/NEJMoa067312 (2007).

3. Black, D.M. et al. The effect of 3 versus 6 years of zoledronic acid treatment of osteoporosis: a randomized extension to the HORIZON-Pivotal Fracture Trial (PFT). [published correction appears in J Bone Miner Res. 2012 Dec;27(12):2612]. J Bone Miner Res.27(2):243-254. DOI: https://doi.org/10.1002/jbmr.1494 (2012).

4. Adami, S. et al. The acute-phase response after bisphosphonate administration. Calcif Tissue Int.41(6):326-331. DOI: https://doi.org/10.1007/BF02556671 (1987).

5. Sato, T. et al. Which symptoms negatively affect the oral health-related quality of life in patients with osteonecrosis of the jaw? Oral Surg Oral Med Oral Pathol Oral Radiol. 130(2):175-180. https://doi.org/10.1016/j.000o.2020.03.051 (2020).

6. Ruggiero, S.L. Guidelines for the diagnosis of bisphosphonate-related osteonecrosis of the jaw (BRONJ). Clin Cases Miner Bone Metab.4(1):37-42 (2007).

7. Malan, J., Ettinger, K., Naumann, E. \& Beirne, O.R. The relationship of denosumab pharmacology and osteonecrosis of the jaws. Oral Surg Oral Med Oral Pathol Oral Radiol.114(6):671-676. DOI: https://doi.org/10.1016/j.000o.2012.08.439 (2012).

8. Ghidini, G. et al. Medication-related osteonecrosis of the jaw: risk factors in patients under biphosphonate versus patients under antiresorptive-antiangiogenic drugs. Minerva Stomatol.66(4):135-140. DOI: https://doi.org/10.23736/S0026-4970.17.04056-0 (2017).

9. Fusco, V., Santini, D., Armento, G., Tonini, G. \& Campisi, G. Osteonecrosis of jaw beyond antiresorptive (bone-targeted) agents: new horizons in oncology. Expert Opin Drug Saf.15(7):925-935. DOI: https://doi.org/10.1080/14740338.2016.1177021 (2016).

10. Oteri, G. et al. Could the Combined Administration of Bone Antiresorptive Drug, Taxanes, and Corticosteroids Worsen Medication Related Osteonecrosis of the Jaws in Cancer Patients? Biomed Res Int.2018:4021952. DOI: https://doi.org/10.1155/2018/4021952 (2018).

11. Khosla, S. et al; American Society for Bone and Mineral Research. Bisphosphonate-associated osteonecrosis of the jaw: report of a task force of the American Society for Bone and Mineral Research. J Bone Miner Res. 22(10):1479-1491. DOI: https://doi.org/10.1359/jbmr.0707onj (2007).

12. Bamias, A. et al. Osteonecrosis of the jaw in cancer after treatment with bisphosphonates: incidence and risk factors. J Clin Oncol.23(34):8580-8587. DOI: https://doi.org/10.1200/JC0.2005.02.8670 (2005).

13. Rizzoli, R. et al. Adverse reactions and drug-drug interactions in the management of women with postmenopausal osteoporosis. Calcif Tissue Int.89(2):91-104. DOI: https://doi.org/10.1007/s00223011-9499-8 (2011).

14. Reid, I.R. \& Cornish, J. Epidemiology and pathogenesis of osteonecrosis of the jaw. Nat Rev Rheumatol.8(2):90-96. DOI: https://doi.org/10.1038/nrrheum.2011.181 (2011).

15. Estilo, C.L. et al. Osteonecrosis of the jaw related to bevacizumab. J Clin Oncol.26(24):4037-4038. DOI: https://doi.org/10.1200/JC0.2007.15.5424 (2008). 
16. Wood, J. et al. Novel antiangiogenic effects of the bisphosphonate compound zoledronic acid. $J$ Pharmacol Exp Ther.302(3):1055-1061. DOI: https://doi.org/10.1124/jpet.102.035295 (2002).

17. Santini, D. et al. Zoledronic acid induces significant and long-lasting modifications of circulating angiogenic factors in cancer patients. Clin Cancer Res.9(8):2893-7 (2003).

18. Vincenzi, B. et al. Serum VEGF levels as predictive marker of bisphosphonate-related osteonecrosis of the jaw. J Hematol Oncol.5:56. DOI: https://doi.org/10.1186/1756-8722-5-56 (2012).

19. Meurman, J.H., Tarkkila, L. \& Tiitinen, A. The menopause and oral health. Maturitas. 63(1):56-62. DOI: https://doi.org/10.1016/j.maturitas.2009.02.009 (2009).

20. Scardina, G.A. \& Messina, P. Oral microcirculation in post-menopause: a possible correlation with periodontitis. 29(2):e1045-1051. DOI: https://doi.org/10.1111/j.1741-2358.2011.00608.x (2012).

21. Catalano, A. et al. Fracture risk assessment in postmenopausal women referred to an Italian center for osteoporosis: a single day experience in Messina. Clin Cases Miner Bone Metab. 10(3):191-194 (2013).

22. Genant, H.K., Wu, C.Y., van Kuijk, C. \& Nevitt, M.C. Vertebral fracture assessment using a semiquantitative technique. J Bone Miner Res.8(9):1137-1148. DOI: https://doi.org/10.1002/jbmr.5650080915 (1993).

23. Rossini, M. et al. Circulating $\gamma \delta T$ cells and the risk of acute-phase response after zoledronic acid administration. J Bone Miner Res.27(1):227-230. DOI: https://doi.org/10.1002/jbmr.521 (2012).

24. Catalano, A. et al. Vitamin D reduces musculoskeletal pain after infusion of zoledronic acid for postmenopausal osteoporosis. Calcif Tissue Int.90(4):279-285. DOI: https://doi.org/10.1007/s00223-012-9577-6 (2012).

25. Reid, I.R., Gamble, G.D., Mesenbrink, P., Lakatos, P. \& Black, D.M. Characterization of and risk factors for the acute-phase response after zoledronic acid. J Clin Endocrinol Metab.95(9):4380-4387. DOI: https://doi.org/10.1210/jc.2010-0597 (2010).

26. Hetland, M.L. et al. Circulating VEGF as a biological marker in patients with rheumatoid arthritis? Preanalytical and biological variability in healthy persons and in patients. Dis Markers.24(1):1-10. DOI: https://doi.org/10.1155/2008/707864 (2008).

27. Misso, G. et al. Evaluation of the in vitro and in vivo antiangiogenic effects of denosumab and zoledronic acid. Cancer Biol Ther. 13(14):1491-1500. DOl: https://doi.org/10.4161/cbt.22274 (2012).

28. Aldridge, S.E., Lennard, T.W., Williams, J.R. \& Birch, M.A. Vascular endothelial growth factor receptors in osteoclast differentiation and function. Biochem Biophys Res Commun.335(3):793-798. DOI: https://doi.org/10.1016/j.bbrc.2005.07.145 (2005).

29. Byrne, A.M., Bouchier-Hayes, D.J.\& Harmey, J.H. Angiogenic and cell survival functions of vascular endothelial growth factor (VEGF). J Cell Mol Med.9(4):777-794. DOI: https://doi.org/10.1111/j.15824934.2005.tb00379.x (2005).

30. Pepe, J. et al. The effect of parathyroid hormone (1-84) treatment on serum bone morphogenetic protein 4 and vascular endothelial growth factor in postmenopausal women with established 
osteoporosis. J Endocrinol Invest.40(6):663-667. DOI: https://doi.org/10.1007/s40618-017-0636-8 (2017).

31. Senel, K. et al. Circulating vascular endothelial growth factor concentrations in patients with postmenopausal osteoporosis. Arch Med Sci. 9(4):709-712. DOI: https://doi.org/10.5114/aoms.2013.36896 (2013).

32. Guise, T.A. Antitumor effects of bisphosphonates: promising preclinical evidence. Cancer Treat Rev.34 Suppl 1:S19-24. DOI: https://doi.org/10.1016/j.ctrv.2008.03.006 (2008).

33. Lipton, A. Emerging role of bisphosphonates in the clinic--antitumor activity and prevention of metastasis to bone. Cancer Treat Rev.34 Suppl 1:S25-30. DOI: https://doi.org/10.1016/j.ctrv.2008.03.008 (2008).

34. Santini, D. et al. Repeated intermittent low-dose therapy with zoledronic acid induces an early, sustained, and long-lasting decrease of peripheral vascular endothelial growth factor levels in cancer patients. Clin Cancer Res.13(15 Pt 1):4482-4486. DOI: https://doi.org/10.1158/1078-0432.CCR-070551 (2007).

35. Ishtiaq, S. et al. The effect of nitrogen containing bisphosphonates, zoledronate and alendronate, on the production of pro-angiogenic factors by osteoblastic cells. Cytokine. 71(2):154-160. DOI: https://doi.org/10.1016/j.cyto.2014.10.025 (2015).

36. Lasco, A. et al. Denosumab Inhibition of RANKL and Insulin Resistance in Postmenopausal Women with Osteoporosis. Calcif Tissue Int.98(2):123-128. DOI: https://doi.org/10.1007/s00223-015-0075-5 (2016).

37. Girolami, I. et al. Denosumab treated giant cell tumour of bone: a morphological, immunohistochemical and molecular analysis of a series. J Clin Pathol.69(3):240-247. DOI: https://doi.org/10.1136/jclinpath-2015-203248 (2016).

38. Shahvegharasl, Z. et al. Effects of cholecalciferol supplementation on serum angiogenic biomarkers in breast cancer patients treated with tamoxifen: A controlled randomized clinical trial. Nutrition. 72:110656. DOI: https://doi.org/10.1016/j.nut.2019.110656 (2020).

39. Zhao, J.W. et al.Vitamin D suppress the production of vascular endothelial growth factor in mast cell by inhibiting PI3K/Akt/p38 MAPK/HIF-1a pathway in chronic spontaneous urticaria. Clin Immunol. 215:108444. DOI: https://doi.org/10.1016/j.clim.2020.108444 (2020).

40. Jamali, N., Song, Y.S., Sorenson, C.M. \& Sheibani, N. 1,25(OH)2D3 regulates the proangiogenic activity of pericyte through VDR-mediated modulation of VEGF production and signaling of VEGF and PDGF receptors. FASEB Bioadv. 1(7):415-434. DOI: https://doi.org/10.1096/fba.2018-00067 (2019).

41. Cardus, A. et al. 1,25-dihydroxyvitamin D3 regulates VEGF production through a vitamin D response element in the VEGF promoter. Atherosclerosis. 204(1):85-89. DOI: https://doi.org/10.1016/j.atherosclerosis.2008.08.020 (2009).

42. Hokugo, A. et al. Increased prevalence of bisphosphonate-related osteonecrosis of the jaw with vitamin D deficiency in rats. J Bone Miner Res. 25(6):1337-1349. DOI: 
https://doi.org/10.1002/jbmr.23 (2010).

43. Ardine, M. et al. Could the long-term persistence of low serum calcium levels and high serum parathyroid hormone levels during bisphosphonate treatment predispose metastatic breast cancer patients to undergo osteonecrosis of the jaw? Ann Oncol.17(8):1336-1337. DOI: https://doi.org/10.1093/annonc/mdl045 (2006).

44. Bedogni, A. et al. Osteomalacia: the missing link in the pathogenesis of bisphosphonate-related osteonecrosis of the jaws? 17(8):1114-1119. DOI: https://doi.org/10.1634/theoncologist.2012-0141 (2012).

45. Sarkarat, F. et al. Platelet-Rich Plasma in Treatment of Zoledronic Acid-Induced Bisphosphonaterelated Osteonecrosis of the Jaws. Trauma Mon.19(2):e17196. DOI: https://doi.org/10.5812/traumamon.17196 (2014).

\section{Tables}

Table 1 - Main clinical features of recruited women at baseline. 


\begin{tabular}{|c|c|c|c|}
\hline & $\begin{array}{l}\text { Zol } \\
(n=18)\end{array}$ & $\begin{array}{l}\text { Controls } \\
(n=10)\end{array}$ & $p$ \\
\hline $\operatorname{Age}(y r)$ & $63.5 \pm 11.1$ & $62.1 \pm 9.2$ & 0.73 \\
\hline Age at menopause (yrs) & $45.3 \pm 5.4$ & $48.6 \pm 4.1$ & 0.12 \\
\hline Time since menopause $(y r)$ & $18.2 \pm 7.4$ & $15.3 \pm 6.5$ & 0.31 \\
\hline$B M I\left(K g / m^{2}\right)$ & $22.85 \pm 2.7$ & $23.1 \pm 1.9$ & 0.79 \\
\hline Current smoking [n(\%)] & $2(10)$ & $1(10)$ & NS \\
\hline Alcohol $\geq$ 3units/day $[n(\%)]$ & $0(0)$ & $0(0)$ & NS \\
\hline $25(\mathrm{OH}) \mathrm{D}(\mathrm{ng} / \mathrm{ml})$ & $44.7 \pm 12.3$ & $43.3 \pm 14.5$ & 0.78 \\
\hline Osteocalcin $(n g / m L)$ & $10.7 \pm 6.9$ & $11.2 \pm 7.1$ & 0.89 \\
\hline CTX $(n g / m L)$ & $0.71 \pm 0.39$ & $0.68 \pm 0.27$ & 0.81 \\
\hline $\operatorname{VEGF}(p g / m L)$ & $121.81 \pm 86.36$ & $106.72 \pm 67.92$ & 0.47 \\
\hline \multicolumn{4}{|l|}{ Bone mineral density } \\
\hline Lumbar spine T-score (SD) & $-2.62 \pm 1.3$ & $-2.71 \pm 1.08$ & 0.81 \\
\hline Lumbar spine BMD $\left(\mathrm{gr} / \mathrm{cm}^{2}\right)$ & $0.75 \pm 0.15$ & $0.74 \pm 0.18$ & 0.79 \\
\hline Femoral neck T-score (SD) & $-2.45 \pm 0.35$ & $-2.32 \pm 0.51$ & 0.43 \\
\hline Femoral neck BMD $\left(\mathrm{gr} / \mathrm{cm}^{2}\right)$ & $0.58 \pm 0.08$ & $0.61 \pm 0.1$ & 0.39 \\
\hline \multicolumn{4}{|l|}{ FRAX score } \\
\hline Major osteoporotic fracture (\%) & $28 \pm 17$ & $25 \pm 14$ & 0.63 \\
\hline Hip fracture (\%) & $12 \pm 9$ & $10 \pm 8$ & 0.56 \\
\hline
\end{tabular}

Data are expressed as mean $\pm S D$ or number (\%) as appropriate. NS= not significant

Zol= Zoledronic acid; $B M I=$ Body Mass Index; 25(OH)D= 25-hydroxyvitamin D; CTX=C-telopeptide of type 1 collagen; VEGF= Vascular Endothelial Growth Factor; $B M D=$ Bone Mineral Density; FRAX= Fracture Risk Assessment Tool.

\section{Figures}




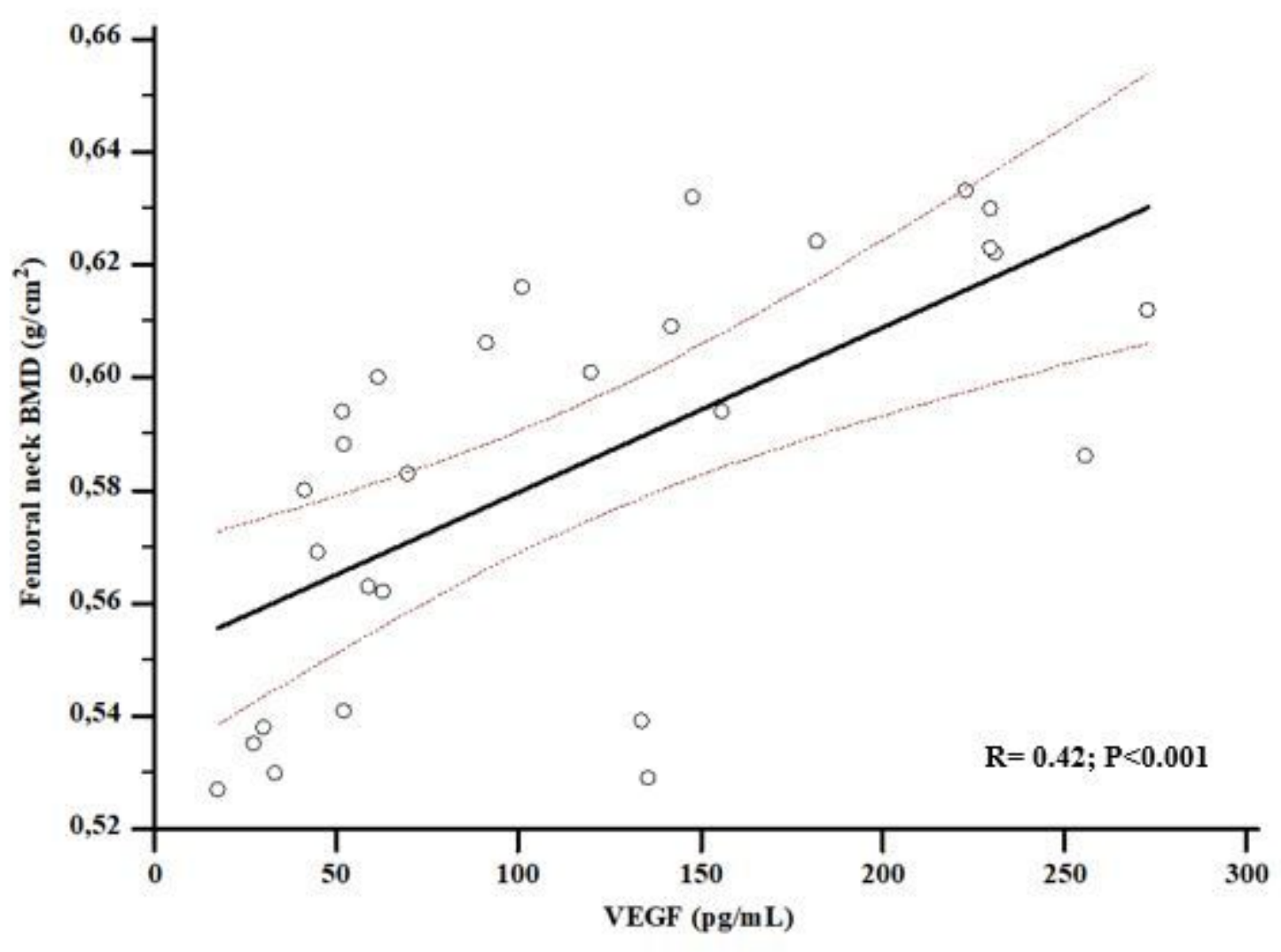

Figure 1

Association between bone mineral density (BMD) at femoral neck and Vascular Endothelial Growth Factor (VEGF) levels at baseline. 


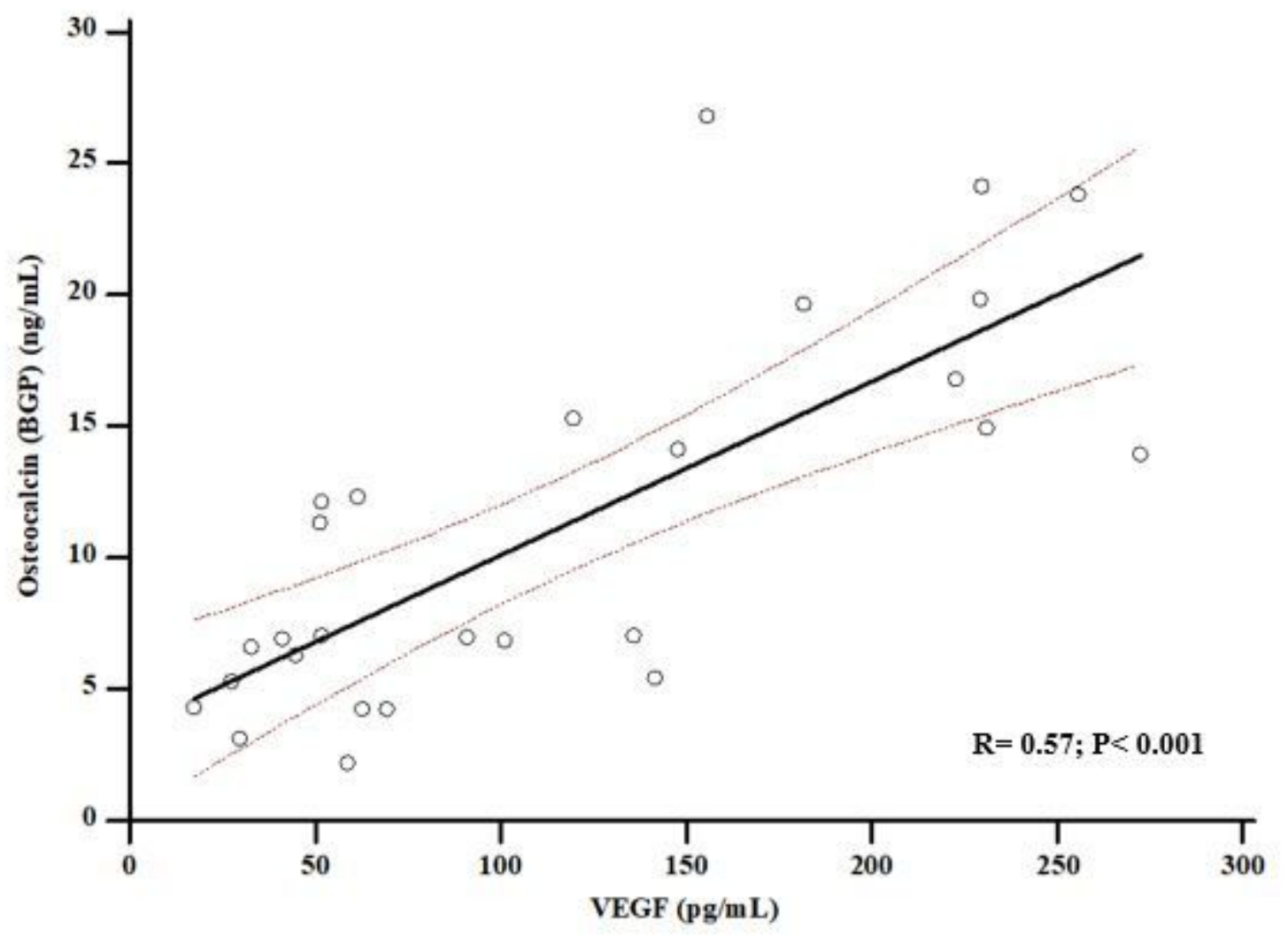

Figure 2

Association between levels of Osteocalcin (BGP) and Vascular Endothelial Growth Factor (VEGF) at baseline. 


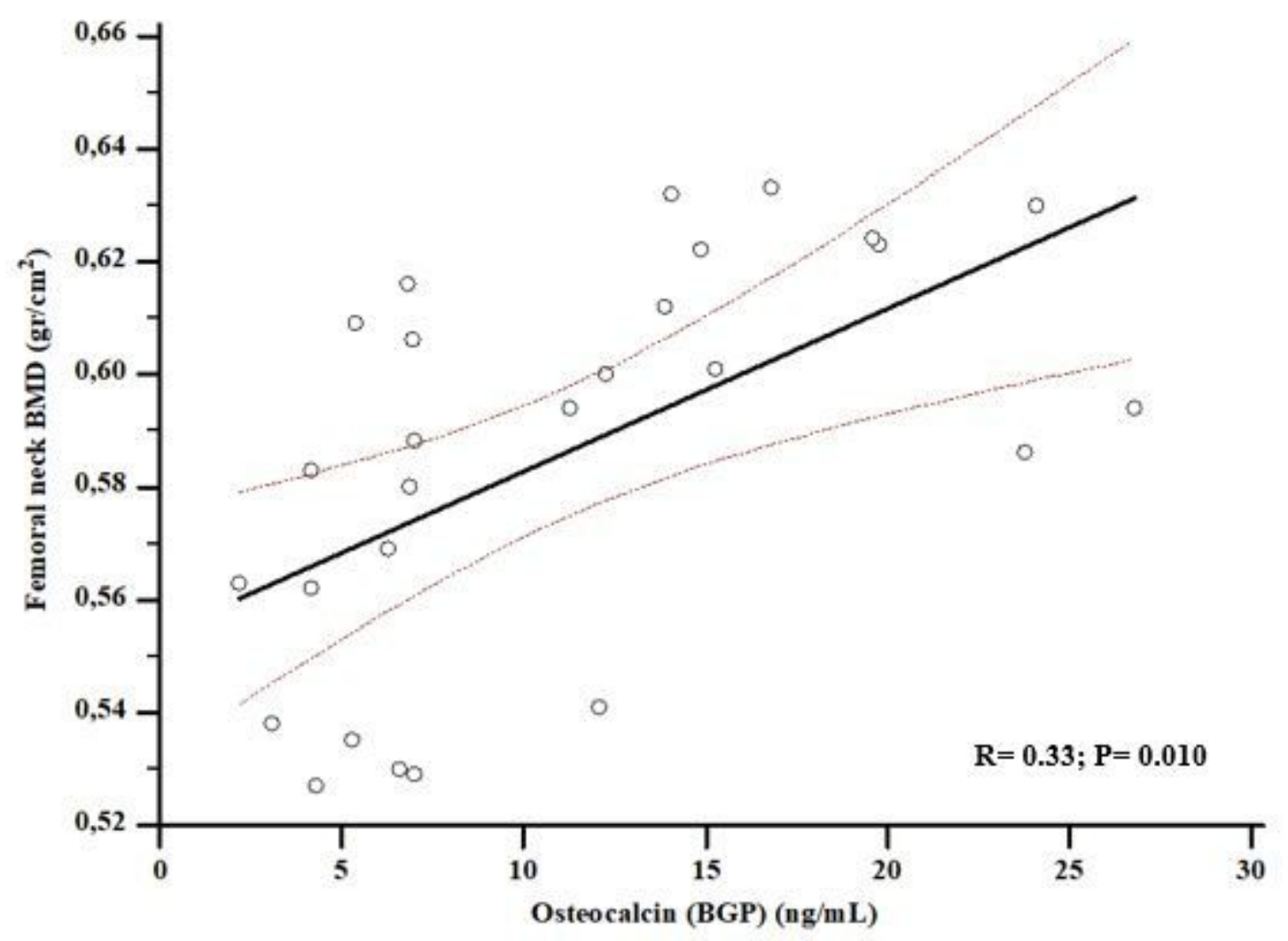

Figure 3

Association between levels of osteocalcin (BGP) and bone mineral density (BMD) at femoral neck at baseline. 


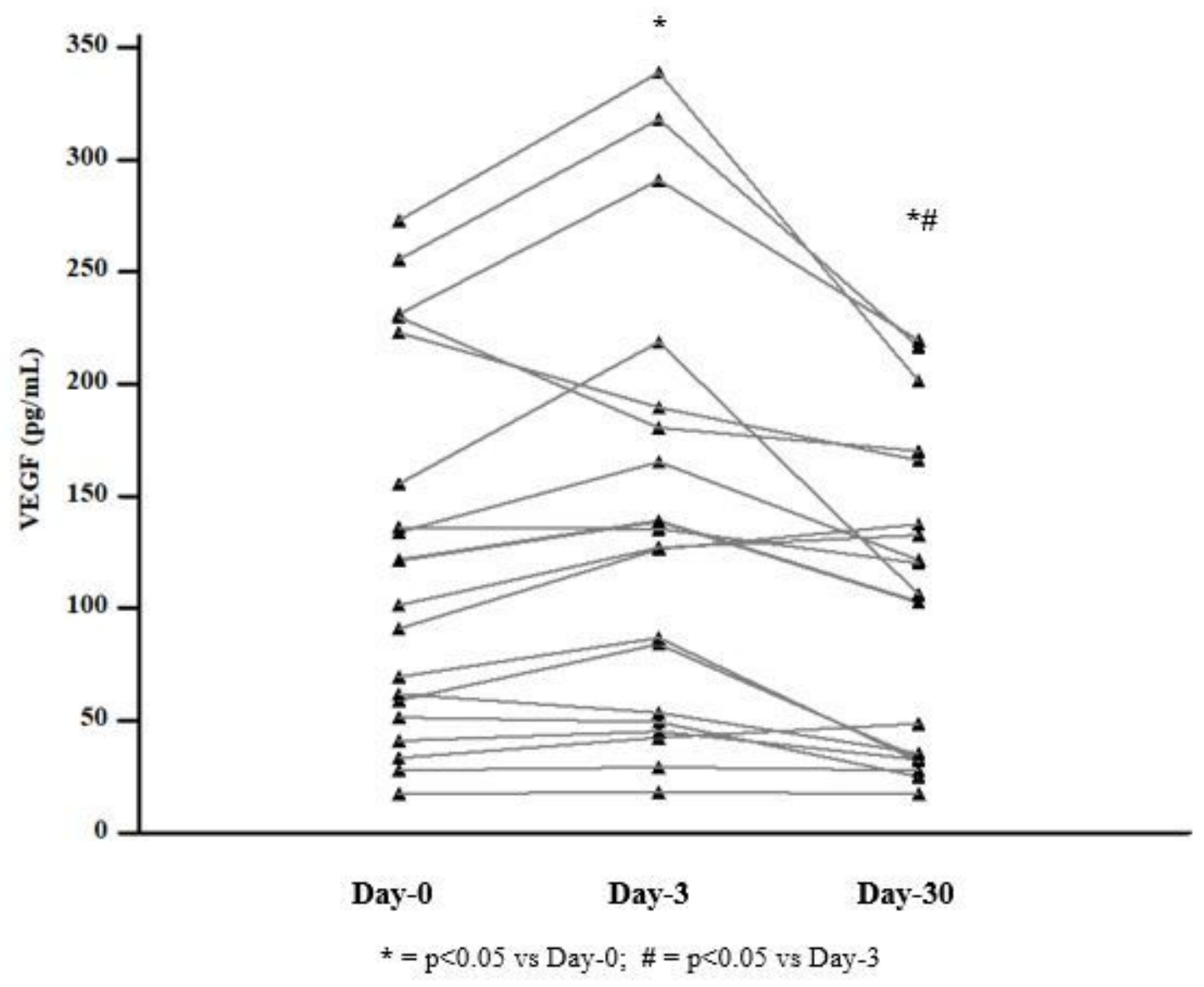

\section{Figure 4}

Levels of Vascular Endothelial Growth Factor (VEGF) at baseline and at day-3 and day-30 after zoledronic acid administration. 


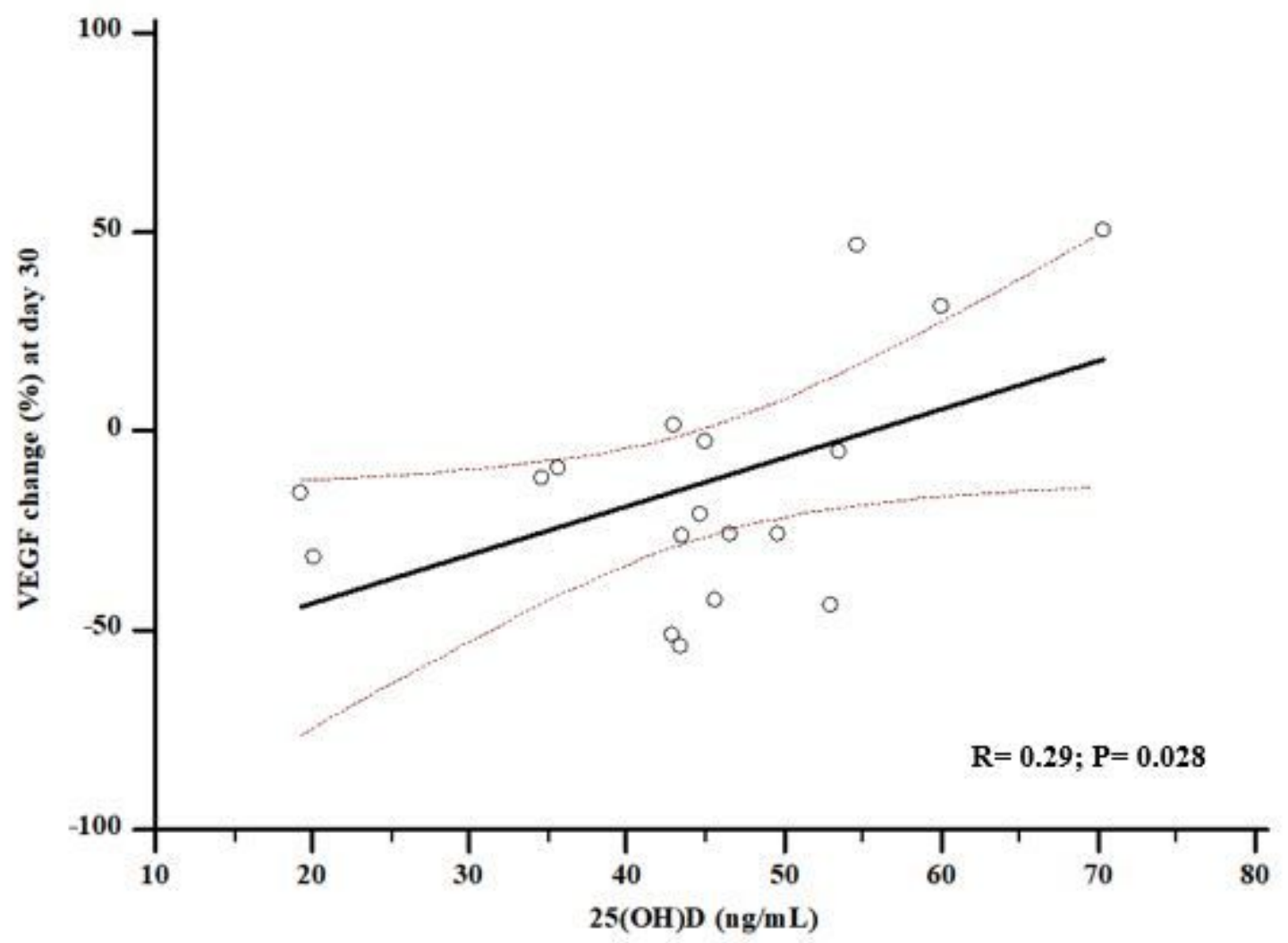

Figure 5

Association between vitamin $D$ status at baseline and percent change of Vascular Endothelial Growth Factor (VEGF) at day-30. 